\title{
Religion im Jugendalter
}

\author{
Ein unsichtbares Phänomen mit offensichtlichen \\ Herausforderungen
}

Thomas Schlag

\section{Jugendliche Unsichtbarkeits-Strategien}

$\mathrm{Zu}$ den zeitunabhängigen Charakteristika des Jugendalters gehört es, die Beweislast dafür tragen zu «dürfen», dass die Zukunft berechtigterweise in hoffnungsvollem Licht erscheint. Der je zukünftigen Generation wird kompensatorisch aufgebürdet, was die erwachsene Gegenwartswelt aus Gründen eigener Fundamentalresignation, überraschungsresistenter Abgeklärtheit oder ritualisierter Alltagsroutine für sich längst ad acta gelegt hat. Aus der Behaglichkeit eigener Arrangements heraus setzt man visionär auf die Jugend, die es nicht nur besser als man selbst haben, sondern die es vor allem besser machen soll.

So verstören jugendliche Gegenreaktionen gegen solche Allmachtsphantasien die Erwachsenenwelt in rationalem und emotionalem Sinn: Wenn «schon in der Jugend» das Gegenteil des Erhofften sichtbar wird, scheint man vor nichts mehr sicher zu sein. So wird der eigentliche Schrecken von «Erfurt» oder «Virgina Tech» nur auf den ersten Blick in den Gewaltakten an Unschuldigen und als Beleg für das latente Gewaltpotential der Gesellschaft angesehen.Vielmehr werden solche Taten vor allem als persönliche finale Angriffe auf die eigenen Wünsche und Zukunftshoffnungen empfunden, die aufgrund der unklar-unsichtbaren Motivlage der Täter das Gefühl fundamentaler Bedrängnis erzeugen. Und so liefert das Bemühen, deviantes und delinquentes Verhalten zu verstehen und zu erklären, vor allem den Beleg für die existentiellen Verunsicherungen der Erwachsenen selbst.

So liegt den vielfältigen Versuchen, die «Generation Jugend» verstehen zu wollen, wesentlich das Motiv zu Grunde, der eigenen Zukunft wenigstens der Hoffnung nach noch einigermassen zuversichtlich entgegenblicken zu können. Die Jugendforschung versucht entsprechend anhand regelmässiger Bulletins über den Stand der Jugend aufzuklären, indem sie schier unendliche Datenmengen über 
deren Lebenslagen, Einstellungen und Zukunftsperspektiven ansammelt, kategorisiert und interpretiert. Und zwischen den Zeilen wird erkennbar, dass es häufig nicht primär um Information, sondern um Prävention, nicht um Verstehen, sondern um Besserungsstrategien, nicht um Erklären, sondern um das prophylaktische Erstellen von Notfallplänen geht. Dabei kann man sich des Eindrucks nicht erwehren, dass die faktischen Befindlichkeiten der Zielgruppe dadurch nur selten auf den Punkt gebracht werden. Es ist davon auszugehen, dass für diese Schwierigkeit einer sachgemässen Annäherung die Befindlichkeiten und Entziehungsstrategien des Jugendalters selbst konstitutiv sind.

Jugendliche Denk- und Lebensvollzüge sind ebenso schwierig eindeutig zu erfassen wie in bestimmte Schemata zu pressen. Selbst den sich authentisch gebenden literarischen Äusserungen, denen man aktuelle Befindlichkeiten abzulesen trachtet, sind in der Regel nur Teilwahrheiten zu entnehmen. Indem sie nicht selten im Habitus stilisierter Betroffenheit oder im Gestus frühreifer Altersweisheit daherkommen, können faktische Lebenslagen eher verschleiert als entziffert werden - und dies im konkreten Fall nicht selten auf bewusste Art und Weise. Jugendliche reagieren in aller Regel hochsensibel auf den Versuch, die eigene Lebensgestaltung für Zukunftshoffnungen der Erwachsenenwelt funktionalisieren zu lassen und machen die eigenen Befindlichkeiten gegenüber den äusseren konventionellen Anforderungen gleichsam unsichtbar. Und dies hat gute Gründe, die in der Lebensentwicklung der Jugendlichen angelegt sind.

In der Phase der Adoleszenz ist prinzipiell von einer starken Ausweitung individueller Wahrnehmungs-, Orientierungs- und Reflexionsfähigkeiten auszugehen. Dies hat einen intensiven Anstieg des Wachsamkeits- und Aufmerksamkeitspotentials bezüglich äusserer Einflüsse und der die Jugendlichen umgebenden Personen zur Folge. Die Such- und Orientierungsdynamik sowie die entsprechend wachsenden kognitiven Kompetenzen führen dazu, dass Jugendliche mit hoher Sensibilität wahrnehmen, was an sie herangetragen wird. Dies kann von den Jugendlichen als innere und äussere Befreiung erlebt, zugleich aber auch mit deutlich steigenden Orientierungsunsicherheiten verbunden werden.

Hinsichtlich des Bindungsverhaltens sind grundlegende Wandlungsprozesse im Gange, insofern an die Stelle der Eltern andere Bezugsgruppen treten, von denen dieselbe Verlässlichkeit, bedingungslose Treue und unzerstörbare Beziehungen sowie prinzipielle Anerkennung erwartet wird. Falls diese jedoch ausbleibt und stattdessen Ablehnungs- undVerlusterfahrungen gemacht werden, ist mit 
intensiven Gegenreaktionen der Jugendlichen sich selbst und der Umwelt gegenüber zu rechnen: Das Ausmass der Verzweiflung ist durchaus mit dem «Verlassenheitsgefühl eines Kindes vergleichbar, das sich von seinen Eltern verlassen fühlt» ${ }^{1}$.

Zugleich konstatiert die entwicklungspsychologisch ausgerichtete Jugendforschung, dass diese in der Pubertätsphase zu realitätsfernen Erwartungen, vorschnellen Urteilen und Selbstüberschätzung neigten: "Allmachtsvorstellungen schlagen, wenn sie auf die Realität treffen, in Sekundenschnelle in Ohnmachtsgefühle um» ${ }^{2}$. Dementsprechend stellen sich gerade in dieser Lebensorientierungsphase eigene Bindungsentscheidungen als in hohem Mass emotional konnotiert dar, die entsprechend im Gestus radikaler Eindeutigkeit - sei es als unbedingte Zuneigung, sei es als massive Distanzierung - ausfallen können ${ }^{3}$.

Deshalb werden in dieser Lebensphase Strategien entwickelt, die eigenen Orientierungsprozesse vor der interessierten und nach «Begleitung» strebenden Öffentlichkeit möglichst umfassend unsichtbar zu halten. Innere Suchbewegungen werden nur höchst selten in einer Weise zum Ausdruck gebracht, dass sie für den «erwachsenen Dialog» anschlussfähig sein können oder sein wollen. Vielmehr scheint es, als ob die individuellen Haltungen gegenüber Erwachsenen gerade nicht versprachlicht werden, sondern aus tiefliegenden Gründen unsichtbar bleiben. So ist etwa für eine konkrete Bindungsentscheidung zwar ein Bündel individueller Kriterien relevant, gleichwohl werden diese Kriterien keineswegs automatisch explizit kommuniziert. Demzufolge ist von einem kommunikativen Moratorium und von der Unsichtbarkeit der eigentlichen Beweggründe für Präferenzen und Ablehnungen $\mathrm{zu}$ sprechen - mindestens gegenüber den bisherigen primären Bezugspersonen. Dies hat seinen vornehmlichen Grund weil die jugendliche Orientierung gleichsam «under construction» ist. Der Versuch der Einflussnahme von aussen auf diese «Bauentwicklung» kann als massive Fundamentalstörung empfunden werden. Die faktischen Lebenslagen, Gefühls- und Gedankendynamiken der Jugendlichen gegenüber denen, die nicht zur unmittelbaren Bezugsgruppe gehören, werden somit aus programmatischen Gründen des Selbstschutzes

\footnotetext{
1 R. Largo, Kinderjahre. Die Individualität des Kindes als erzieherische Herausforderung, München ${ }^{13} 2007,147$.

2 Ebd., 144.

3 Vgl. E. Hauschildt, Gewalt als religiöses Gruppenphänomen. Religionssoziologie, Praktische Theologie und die Einsichten aus der Gruppensoziologie, in: F. Schweitzer (Hg.), Religion, Politik und Gewalt. Kongressband des XII. Europäischen Kongresses für Theologie 18-22. September 2005 in Berlin, Gütersloh 2006, 759-775.
} 
unsichtbar gemacht - und dies im intuitiven Wissen, dass man in dieser Lebensphase besonders angreifbar und gerade deshalb schutzund rückzugsbedürftig ist.

Was Jugendliche fühlen, machen und wofür sie sich jeweils entscheiden, wird in der Regel in diesem Lebensalter vornehmlich mit denjenigen Personen kommuniziert, denen man sich jetzt verbunden fühlt und denen man gegenwärtig vertraut: «In der Gleichaltrigengruppe ist ein Austausch von Sichtweisen und Gefühlen unter Personen gleichen Rangs und mit vergleichbarem Erfahrungshorizont möglich, weil keine überlegene Person in kulturell festgelegtes Wissen und Können einführt» ${ }^{4}$. Es trifft folglich keineswegs zu, dass sich Jugendliche prinzipiell der Kommunikation selbst über wesentliche Erfahrungen, Gefühle und Gedanken verschliessen. Vielmehr zeigen erste Analysen des Welt-Umgangs in Chatrooms, virtuellen Welten, aber auch des Handygebrauchs und interaktiver Computerspiele ein erhebliches Mitteilungsbedürfnis und sehr spezifische Kommunikationskompetenzen ${ }^{5}$ - auch wenn einstweilen unklar ist, ob hinter diesen Formen der Kommunikation vornehmlich die ichbezogene Intention steht, Informationen «über sich selbst» weiterzugeben oder ob es tatsächlich um echten kommunikativen Austausch und Erkenntnisgewinn geht.

Diese Unsichtbarkeitsstrategie lässt sich nun auch für den Bereich religiöser Haltungen und Einstellungen im Jugendalter feststellen, denen man sich - nicht selten aus denselben oben genannten Hoffnungs- und Präventionsgründen - durch unterschiedliche Forschungsansätze und Messverfahren anzunähern versucht. Je nach Ausrichtung des konkreten Forschungsdesigns wird dabei prinzipiell von bestimmten Hypothesen im Blick auf Religion selbst und ihre Manifestationsformen ausgegangen. Dies kann sich in der Rede vom Ultimaten darstellen, auf ein funktionales Verständnis von Religion bezogen sein oder in der Profilierung von Religion als einer mit

${ }^{4}$ K. Hurrelmann, Einführung in die Sozialisationstheorie, Weinheim/Basel ${ }^{8} 2002$, 241.

5 H.Theunert. Medien als Orte informellen Lernens im Prozess des Heranwachsens, in: Sachverständigenkommission Zwölfter Kinder- und Jugendbericht (Hg.), Kompetenzerwerb von Kindern und Jugendlichen im Schulalter, München 2005, 175 300; A. Barsch/J. Zinnecker, Jugend, Jugendszenen und Medien. Merkmale heutiger Jugendkulturen, in: H.-D. Kübler/E. Elling (Hg.), Wissensgesellschaft. Neue Medien und ihre Konsequenzen, Bonn 2004, 553-576; B. Herzig/S. Grafe, Digitale Medien in der Schule. Standortbestimmung und Handlungsempfehlungen für die Zukunft. Studie zur Nutzung digitaler Medien in allgemein bildendenden Schulen in Deutschland, Bonn 2006. 
biographischen Sinnfragen verbundenen anthropologisch nahe liegenden Orientierungsbewegung bestehen.

Einschlägige Untersuchungen lassen sich so deuten, dass die «Religiosität» von Jugendlichen nach wie vor ein prinzipiell weit verbreitetes und intensiv gelebtes Phänomen darstellt ${ }^{6}$. Dies wird nicht nur an der hohen Prozentzahl von Jugendlichen deutlich, die für sich selbst an Gott oder eine höhere, transzendente Macht glauben, von einer göttlichen Schöpfung und Bewahrung der Welt und ihres eigenen Lebens ausgehen, sondern auch an deren religiösen Vollzügen wie etwa dem Gebet und selbst der Teilnahme an kirchlicher Praxis. Auch die Zugehörigkeit zu der konfessionellen Gemeinschaft, der sie aufgrund ihrer Taufe angehören, wird von ihnen weitaus weniger in Frage gestellt als dies landläufig angenommen wird. Entsprechende Einzelergebnisse neuerer Studien weisen zudem darauf hin, dass bei Jugendlichen im Blick auf ihre individuelle Weltsicht und Handlungsorientierung religiöse Deutungsangebote und Orientierungen präsent sind, die sie im Lauf ihrer Sozialisation erfahren und erlebt haben. Prägekräfte können hier die eigene Familie, vorschulische Erfahrungen mit Religion in Kindertagesstätte und Kindergarten, schulischer und kirchlicher Religionsunterricht, die Teilnahme an Angeboten kirchlicher Jugendarbeit ${ }^{7}$ oder auch Medieninformationen sein, denen sie in ihrer Alltagswelt begegnen.

Allerdings besteht bei einer Fokussierung auf diese traditionellen Bezugspunktevon Religion die Gefahr,konkrete religiösePhänomene bei Jugendlichen sogleich auf bestimmte dogmatische Standards oder deren institutionellen Bezug hin zu erfragen bzw. zu «lesen». Dies führt dies nicht selten entweder zu problematischen Stufenmodellen hinsichtlich angeblicher religiöser Entwicklungsfortschritte oder $\mathrm{zu}$ vergleichsweise groben Ergebnissen hinsichtlich des faktischen Vorhandenseins oder Nichtvorhandenseins von Religion in der entsprechenden Altersgruppe. So kann man sich des Eindrucks nicht erwehren, dass die tatsächliche religiöse Gedankenwelt Jugendlicher im Gegenüber zu den klassischen Glaubensstandards in solchen Statistiken und Trends seltsam blass bleibt. Wenn sich Jugendliche andererseits dann doch intensiv über «meinen Glauben» äussern, erfolgt dies nicht selten in dogmatischen Chiffren einer entsprechenden fun-

\footnotetext{
${ }^{6}$ Vgl. 15. Shell-Studie Jugend 2006. Frankfurt a.M.; für den schweizerischen Zusammenhang auch R.J. Campiche, Die zwei Gesichter der Religion. Faszination und Entzauberung, Zürich 2004.

7 K. Fauser/R. Münchmeier/A. Fischer, Jugendliche als Akteure im Verband. Ergebnisse einer empirischen Untersuchung der Evangelischen Jugend, Opladen 2006.
} 
damentalistischen Bezugsgruppe, die allerdings keineswegs automatisch mit den innersten individuellen Beweggründen kongruent sein müssen. Zudem ist die Frage, ob hinter einer Reihe von Umfragen nicht vor allem das Motiv steht, klären zu wollen, wie es um den Glauben der Zukunft und die Kirche der Freiheit wohl bestellt sein wird, wenn die jetzige Jugendgeneration ins zahlungspflichtige Alter kommt. Damit droht dann aber aus kirchlich-strategischen Gründen die Abblendung eines weiten Bereichs jugendlicher Religiosität.

Nimmt man hingegen die Perspektive "gelebter Religion»" ernst, so erscheint eine Annäherung an vorhandene Phänomene über den Begriff individueller Religiosität weiterführend, da sich damit individuelle Wahrnehmungen und Vollzüge von Religion besser in ihrer je individuellen Eigengestalt und den entsprechenden Ausdrucksformen in den Blick nehmen lassen als durch deduktiv vorausgesetzte Typologisierungen.

Die religionspädagogische Grundfrage lautet, wie sich Annäherungen an jugendliche Religiosität so denken lassen, dass die beschriebenen Lebenslagen der Jugendlichen in ihrem meist unsichtbaren Eigen-Sinn und Eigen-Recht anerkannt werden und gleichwohl Orientierungsmöglichkeiten im Modus persönlichkeitsorientierter religiöser Bildung eröffnet werden. Für einen konstruktiven Umgang mit aktuellen Distanzierungs- und Entfremdungstendenzen Jugendlicher im Blick auf Kirche, traditionelle Dogmen und die entsprechenden ethischen Normvorstellungen sei im Folgenden an die von Luckmann unter dem Signum der «unsichtbaren Religion» vorgenommene religionssoziologische Deutung von Religion und Religiosität im Kontext der Moderne erinnert.

\section{2. «Unsichtbare Religion» heute}

Luckmanns Grundthese von der Ersetzung der institutionell spezialisierten Religion durch eine grundsätzlich neue Sozialform von Religion ist als Deutungsperspektive des gegenwärtigen Verhältnisses von Jugend und Religion nach wie vor ausgesprochen hilfreich. Auch wenn sich der Terminus «unsichtbar» in der einschlägigen Schrift bis auf den Titel und einer Nebenbemerkung zur sichtbaren und unsichtbaren Kirche ${ }^{9}$ nicht findet, ist doch der gesamte Essay von dort her konzipiert. Im Anschluss an Durkheims

8 A. Grözinger (Hg.), «Gelebte Religion» als Programmbegriff systematischer und praktischer Theologie, Zürich 2002.

9 Vgl. Th. Luckmann, Die unsichtbare Religion, Frankfurt a.M. 1991, 57. 
und Webers Perspektiven auf das Schicksal des Menschen in der modernen Gesellschaft wird Religion in einem funktionalen Sinn als entscheidender Schlüssel zum Verständnis gesellschaftlicher und individueller Entwicklungsprozesse angesehen und das Problem individueller Daseinsführung in der modernen Gesellschaft als eine primär «religiöse» Frage signiert.

Im Unterschied zur gängigen Säkularisierungsthese geht Luckmann für die moderne westliche Gesellschaft nicht vom Auszug, sondern von Transformationsprozessen der Religion hin zu individueller Religiosität und damit von einer entsprechenden Pluriformität religiös konnotierter Handlungspraxen aus. Damit wird der schon Anfang der 60er Jahre behauptete Auszug von Religion aus der Gesellschaft von Luckmann negiert und die These einer umfassenden Säkularisierung individueller und gemeinschaftlicher Lebenszusammenhänge in grundsätzlichem Sinn relativiert. Die Grundfrage Luckmanns lautet dementsprechend, «ob in der modernen Gesellschaft andere sozial objektivierte Sinnstrukturen als die der traditionellen institutionalisierten religiösen Dogmen die Funktion der Integration alltäglicher Routinen und der Legitimation ihrer Krisen erfüllen $»^{10}$.

Wenn also die explizite Zustimmung zu traditionellen Glaubensinhalten und die sichtbare Identifizierung mit Christentum und Kirche unverkennbar massiv geschwunden sind, so bahnt sich Religion Luckmann zufolge gleichwohl in der ausdifferenzierten Gesellschaft ihre eigenen Wege, indem sich Individuen aus eigenem Antrieb passende Weltansichten und einen subjektiv relevanten «heiligen Kosmos» erschaffen. Luckmann macht somit die Unterscheidung zwischen der Behauptung eines prinzipiellen Nichtmehrvorhandenseins von Religion und ihrem nicht (mehr) im bisherigen Sinn sichtbaren Vorhandensein stark.

Unsichtbar ist Religion in dem Sinn geworden, dass sich die individuellen Suchbewegungen transzendente Anhaltspunkte gesucht haben und suchen, die nicht mehr in den bisherigen dogmatischen Kategorien artikuliert werden, mithin nicht mehr den bisherigen Ausdrucksgestalten traditioneller monopolhafter Religion entsprechen und folglich auch nicht mehr in der gewohnten Weise für die Deutung biographischer Sinnzusammenhänge zu Rate gezogen werden: "So kann der Verlust der Sichtbarkeit der Religion - als gesellschaftlicher Struktur, wie als kultureller Inhalt - dazu führen, dass die persönliche Identität zur letzten Instanz

\footnotetext{
10 Ebd., 61.
} 
der Organisation des Religiösen wird» ${ }^{11}$. Individuelle Religiosität lebt von subjektiven Reflexionsvorgängen, privaten Deutungen und Wahlentscheidungen, die weitgehend unabhängig von den offiziellen Modellen der Sinndeutung getroffen werden. Die neue Sozialform der Religion ist folglich dadurch charakterisiert, «dass potentielle Konsumenten einen direkten Zugang zum Sortiment der religiösen Repräsentationen haben ${ }^{12}$ und sich aus dem Warenlager des Heiligen Kosmos eigenhändig ein klar umschriebenes privates System von letzten Bedeutungen zusammenbauen. Indem die traditionellen Institutionen ihre sozialisatorische Kraft mehr und mehr verloren haben und damit die hergebrachten Formen und Inhalte religiöser Kommunikation deutlich zurückgetreten sind, zieht sich Religion in das Refugium privater Symbolwelten zurück, wo sie sich nun in einer von aussen her kaum dechiffrierbaren geradezu unsichtbaren Weise manifestiert.

Dass Religion per se und trotz nachlassender Bindungskräfte nach wie vor ein höchst lebhaftes Phänomen ist, hat Luckmann zufolge seine Ursache in der anthropologischen Verfasstheit des Menschen selbst, der existentiell und jenseits aller historisch-kulturellen Entwicklungen auf Religiosität hin angelegt ist. Im Sinn von Religion als kommunikativem Konstrukt entsteht durch Faceto-Face-Kommunikation und soziale Interaktion ein Bewusstsein für die Angewiesenheit auf andere. Indem über kleine, mittlere und grosse Transzendenzen im Modus von Sprache, rituellen Akten und Symbolen die biologische Natur transzendiert wird und sich ein heiliger Sinnbereich sowie die entsprechende «Weltansicht» herauskristallisiert, zeigen sich individuelle Transzendierungsvorgänge per se in ihrem religiösen Charakter: «Im Prozess der Sozialisation wird eine historische Weltansicht verinnerlicht. Das objektive Sinnsystem wird in eine subjektive Wirklichkeit umgewandelt» ${ }^{13}$. Die analytische Pointe besteht darin, dass Luckmann zufolge gerade die Entwicklung zu kirchlichem Expertentum und institutionalisierten religiösen Normen dazu geführt hat, dass sich das System subjektiver Präferenzen von den normativen Grundlagen mehr und mehr ablöst: «Anliegen von 〈letzter» Bedeutung, wie sie im 〈offiziellen» Modell festgelegt sind, können im Prinzip in gewohnheitsmässige und unzusammenhängende Erfüllungen oder Teilerfüllungen oder

${ }^{11}$ H. Knoblauch, Die Verflüchtigung der Religion ins Religiöse. Thomas Luckmanns Unsichtbare Religion, in: Luckmann, Unsichtbare Religion, s. Anm. 9, $32 \mathrm{f}$.

${ }^{12}$ Luckmann, Unsichtbare Religion, s. Anm. 9, 146.

13 Ebd., 109. 
Verweigerungen spezifisch religiöser Pflichten umgewandelt werden, deren 〈heilige` Qualität nurmehr nominell gilt» ${ }^{14}$.

Was Luckmann im historischen Zusammenhang des gesellschaftlich bedingten Strukturwandels von der sichtbaren zur unsichtbaren Religion formuliert hat, lässt sich in aktueller Hinsicht als hilfreiche Beschreibung des religiös konnotierten Institutionen- und Generationenproblems ansehen und seine weitgreifende historische Analyse kann mikroskopisch auf das Verhältnis der jungen zur erwachsenen Generation angewandt werden: «Die alltäglichen Sorgen der Väter sind nicht mehr die der Söhne, und viele Sorgen der Söhne sind ihren Vätern unbekannt» ${ }^{15}$. Auf die gegenwärtige Debatte über Religion übertragen bedeutet dies, dass die These von der Wiederkehr von Religion schon deshalb problematisch ist, weil damit suggeriert wird, dass diese zwischenzeitlich tatsächlich einmal verschwunden gewesen sein soll und nun in gleicher Gestalt wie zuvor erneut auf die Bühne trete bzw. treten könne.

Vielmehr ist Luckmanns Analyse zuzustimmen, dass sich mindestens für den Bereich westlicher Gesellschaften ein fundamentaler und geradezu unumkehrbarer Veränderungsprozess der bisherigen Manifestation von Religion ereignet hat, der seinerseits selbst durch pädagogische Massnahmen ${ }^{16}$ kaum aufzuhalten sein dürfte. Die neue Sozialform von Religion, so Luckmann in einem späteren Nachtrag zu seinen Ausführungen, zeichnet sich weniger durch das aus, was sie ist, als vielmehr durch das, was sie nicht ist: «durch das Fehlen allgemein glaubwürdiger und verbindlicher gesellschaftlicher Modelle für dauerhafte, allgemein menschliche Erfahrungen der Transzendenz» ${ }^{17}$.

Hiesse dies aber dann nicht auch, dass die neue Sozialform von Religion eben nicht nur ein Moratorium darstellt, mit dessen gutem Ende irgendwann wieder $\mathrm{zu}$ rechnen ist, sondern die zukünftige alternativenlose neue Form von Religion schlechthin als «eine «logische> Folge ... des hohen Grades der funktionalen Differenzierung der Sozialstruktur» ${ }^{18}$, so dass jede Form der Intervention von vorneherein zum Scheitern verurteilt wäre? Ist dies im Blick auf «ugend und Religion» somit der status quo, hinter den es kein Zurück gibt?

\footnotetext{
${ }^{14}$ Luckmann, Unsichtbare Religion, s. Anm. 9, 115.

15 Ebd., 123.

16 Ebd., 116.

17 Ebd., 182.

18 Ebd., 179.
} 
Wäre es also in Abwandlung eines Marquardschen Diktums das Beste, die Jugend nicht verändern zu wollen, sondern sie einfach zu verschonen und die individuelle und gesellschaftliche Entwicklung als Normativität des Faktischen in ihrer Eigendynamik vorbehaltlos anzuerkennen? Oder ist gerade aufgrund der jugendlichen hochsensiblen Wahrnehmungskompetenz erst recht missionarische Aktivität und religiöse Gruppenbildung angesagt - immerhin leben die entsprechenden Angebote von genau dieser Einsicht und Strategie. Auf dem Hintergrund der Luckmannschen Analyse sind jedenfalls alle kirchlichen Versuche, die Bindungswirkungen der eigenen Institution durch möglichst attraktive Angebote zu erhöhen und Jugendliche dafür zu begeistern, kritisch wahrzunehmen und einzuschätzen.

Gleichwohl lässt sich an die Luckmannschen Beschreibungen durchaus positiv anknüpfen, indem im Anschluss an ihn von folgender Annahme ausgegangen wird: Die immer wieder konstatierten Verflüchtigungstenzenden sichtbarer Religion samt der entsprechenden Kommunikationsresistenz spiegeln gerade die Ernsthaftigkeit individuellen jugendlichen Bemühens um Freiheit und Autonomie auch im Bereich religiöser Orientierung wider. Vermögen Jugendliche ihren Suchbewegungen keinen explizit kirchlich gebundenen Sinn zu verleihen, so ist dies nicht als absichtsvolle Renitenz zu interpretieren, sondern als Ausdruck für einen eigenständigen hochdynamischen Suchprozess, der in dieser Phase eigener Entscheidungsfindung eben aus tief liegenden Gründen kaum Konzessionen gegenüber der «älteren Generation» und den von ihnen repräsentierten Institutionen samt entsprechender Botschaften erlaubt. Dass man sich notwendigerweise eigene Wege suchen muss, hängt aber auch damit zusammen, dass von den erwachsenen «Bildungsagenten» immer seltener religiöse Deutungsangebote überliefert werden - in Luckmanns Diktion: dass die notwendige Face-to-Face-Kommunikation in Fragen der Religion schlichtweg verweigert wird. So gilt etwa: «Die Sehnsucht nach einer christlichen Erziehung der eigenen Kinder ist oft genug die Kehrseite der religiösen Ungewissheit der Eltern. Diese wünschen sich für ihren Nachwuchs eine Klarheit und Unbedingtheit, die ihnen selbst abgeht» ${ }^{19}$.

Die Zusammenstellung eines höchst privaten religiösen Gemisches samt der entsprechenden Ritualisierungen im Gegenüber zu den vorgegebenen Orientierungen liegt demzufolge in einer Flucht-

${ }^{19}$ J.H. Claussen, Zurück zur Religion. Warum wir vom Christentum nicht loskommen, München 2006, 252. 
bzw. Suchlinie, die einerseits mit den entwicklungspsychologischen Prozessen des Jugendalters, andererseits unmittelbar mit den ausfallenden religiösen Sozialisationsprozessen zusammenhängt. Dies ist im Übrigen auch der Grund dafür, dass sich die Fragen einer «Kindertheologie» gegenwärtig wesentlich intensiver behandelt werden als etwa Aspekte einer «Jugendtheologie».

Zudem lässt sich von Luckmann her das Phänomen deuten, warum Jugendliche auch in dieser Frage am ehesten mit Gleichgesinnten ihrer eigenen Gruppe über ihre eigenen religiösen Fragen kommunizieren: denn auch in religiösen Suchbewegungen sind nicht die Vertreter des Althergebrachten die entscheidenden Orientierungspersonen, sondern diejenigen in der gleichen Lebenslage, mit denen man sich aufgrund der gemeinsamen Befindlichkeit solidarisch fühlt. Die Privatheit ist folglich nicht als solipsistische, sondern in der Regel eher als eine durch Reziprozität gewährleistete, stabilisierte und geschützte Privatheit zu lesen. Wenn nun aber von einer solchen jugendlichen Dynamik auszugehen ist, stellt sich unweigerlich die Frage danach, wie damit sinnvollerweise umgegangen werden bzw. was dies für Kommunikationsprozesse im Rahmen religiöser Bildungsangebote bedeuten kann.

\section{Offensichtliche Herausforderungen}

Die entscheidende Aufgabe im Umgang mit der unsichtbaren Religion im Jugendalter kann nicht darin bestehen, Strategien zum Einbruch in die individuelle Moratoriumsphase zu entwickeln, sondern sich einerseits für den Fall der neuerlichen Kontaktaufnahme von den Jugendlichen aus bereit zu halten, andererseits diese Bereitschaft zur Kontaktaufnahme durch entsprechende Kommunikations- und Partizipationsanreize in angemessener Weise zu initiieren.

Sensible Zeitgenossen profilieren entsprechend die Aufgabe des Erwachsenen in Bildungszusammenhängen als die eines Begleiters, der seine Aufmerksamkeit und prinzipielleVerfügbarkeit für den Fall signalisiert, in dem der Jugendliche selbst zur Kontaktaufnahme bereit ist. Die pädagogische Kunst besteht darin, bei der dem Jugendlichen entsprechenden Gelegenheit schlichtweg präsent und ansprechbar zu sein. Dazu gehört es, durch konkrete Gesprächsangebote $\mathrm{zu}$ signalisieren, dass sich diese Kontaktaufnahme lohnen könnte. Konkret ist etwa im Fall der professionellen Beziehung zwischen Lehrer und Schüler offen zu kommunizieren, worin die Aufgabe des Lehrers aufgrund seiner eigenen Rolle besteht und in welchem Sinn 
diese Rollenzuschreibung notwendigerweise mit bestimmten Orientierungsverpflichtungen verbunden ist. Dies mag für den Jugendlichen sperrig wirken, aber gerade eine offene Benennung der faktischen Rollenaufgabe eröffnet am ehesten die Chance auf jugendliche Akzeptanz und hält die Option jugendlicher Bindungsbereitschaft offen. Eine Selbstaufgabe oder Unkenntlichmachung der eigenen Rolle samt entsprechender Anbiederungssignale dürfte hingegen auf Seiten des Jugendlichen «on the long run» die grössten Irritationen und Distanzierungsbewegungen auslösen.

Innerhalb der Rollenausübung jedoch ist es zugleich notwendig, diejenigen Dimensionen aufzuzeigen, die der Rolle und Person des Lehrenden eine inhaltlich gestützte Legitimation verschaffen. Insofern lebt beispielsweise die zugeschriebene Rolle des Fremdenführers davon, dass dieser tatsächlich die Kompetenz hat, die Ausweitung jugendlicher Perspektiven auf «fremdes» Gebiet als attraktiv erscheinen zu lassen. Schliesslich wird die Rolle des empathisch-solidarischen Begleiters dann als legitim eingeschätzt, wenn sie anhand stimmiger Kommunikations- undVerhaltensmuster plausibilisiert wird.

Das vornehmste Ziel der Beschäftigung mit jugendlicher Religion besteht jedoch in der verstärkten Wahrnehmung der Suchbewegungen und Irritationspotentiale ${ }^{20}$. Kirchliches Bildungshandeln bekommt überhaupt erst seine Adressaten und deren Lebenswirklichkeiten angemessen in den Blick, wenn sie von vorne herein kirchlich gebundenen christlichen Glauben einerseits und individuelle Religiosität andererseits in ihrer faktischen und historisch begründeten Unterschiedenheit in den Blick zu nehmen versteht.

Notwendig ist folglich eine erhöhte Sensibilität für Erscheinungsformen von Religiosität im Jugendalter jenseits der klassischen Manifestationsformen. Ein solches Plädoyer für die verstärkte Aufmerksamkeit auf die unsichtbare Seite individueller privater Religiosität mag paradox erscheinen, da sich doch das Unsichtbare per definitionem nicht von selbst erschliesst, sondern gerade verschlossen bleibt.

Nun können sich gerade Nebenbemerkungen en passant, die unterschiedlichen Ritualpraxen und Symbolisierungen als Manifestation von Sinnsuche, Bindungsbereitschaft und Distanznahme am Ort des Individuums und seiner Gruppe zeigen. Diese weisen in ihrer sozialen Ausdrucksgestalt darauf hin, dass nicht alles an der un-

${ }^{20}$ Vgl. U. Schwab,Verhältnis der Generationen, in: R. Lachmann/G. Adam/M. Rothgangel (Hg.), Ethische Schlüsselprobleme. Lebensweltlich - theologisch - didaktisch, Göttingen 2006, 267-285. 
sichtbaren Religion unsichtbar und nicht alles Private der äusseren Wahrnehmung verschlossen bleiben muss.

Solche Versuche der Wahrnehmung jugendlicher Ausdrucksformen von Religiosität können im positiven Fall sogar zu der Rückkoppelung führen, dass Jugendliche sich gerade dann in ihrer je individuellen und gruppenorientierten Wirklichkeitskonstruktion als wahrgenommen und anerkannt empfinden können. So besteht die pädagogische Kunst darin, Jugendliche gerade in den entindividualisierenden kulturellen Kontexten ihrer Lebenswelten Aufmerksamkeit zu signalisieren und darauf zu hoffen, dass sie darauf - bei Gelegenheit - zurückkommen werden. Insofern ist schon dieser Versuch geschärfter und aufmerksamer Wahrnehmung keinesfalls zu unterschätzen - auch wenn dessen mögliche positive Konsequenzen einstweilen scheinbar ohne sofortige sichtbare Folgen bleiben.

Von hier aus stellt sich allerdings die weitere inhaltsbezogene Frage, ob sich in dieser angebotenen Face-to-Face-Kommunikation das «traditionelle Universum religiöser Ideen»" so plausibilisieren lässt, dass es als bedeutsame Grösse für die Entwicklung individueller Weltansicht wahrgenommen wird bzw. dass eine «entfaltete und reflektierte Religiosität» ${ }^{22}$ von den Jugendlichen selbst als bedeutsamer Aspekt in entsprechenden Orientierungsprozessen erkennbar und erlebbar wird. Ist also doch eine «Umkehrung» der Abwendung von klassischen Institutionen und deren Sinnangeboten denkbar?

Folgt man Luckmanns Analyse, dann nur dann, wenn sich diese klassischen Manifestationen selbst in neuer Weise als attraktive sinnstiftende Angebote zeigen und die Erwachsenen selber zum partnerschaftlichen Umgang zwischen den Generationen und der «Anerkennung von Jugendlichen als Subjekten in Kirche und Gesellschaft» ${ }^{23}$ beitragen. Dies setzt zuallererst voraus, dass die «erwachsenen» Sozialisationsagenten für sich selbst religiöse Sprachfähigkeit entwickeln und für sich durchbuchstabieren, welche existentiellen Bezüge sie zwischen Religion, christlicher Überlieferung und der Institution Kirche herzustellen vermögen und dies entsprechend transparent machen. Unterbleibt dies, ist hingegen mit Folgendem zu rechnen: «Was die Väter predigen, aber nicht vorexerzieren, wird von den Söhnen eher als ein rhetorisches System denn als ein System letzter〉 Bedeutung verinnerlicht. Im

\footnotetext{
${ }^{21}$ Luckmann, Unsichtbare Religion, s. Anm. 9, 71.

${ }^{22}$ J. Kunstmann, Religiosität und Bildung. Pädagogisch-bildungstheoretische Perspektiven, in: H.-F.Angel u.a. (Hg.), Religiosität. Anthropologische, theologische und sozialwissenschaftliche Klärungen, Stuttgart 2006, 162

${ }^{23}$ F. Schweitzer, Religionspädagogik. Gütersloh 2006, 231.
} 
äussersten Fall - unter der Annahme, dass das «offizielle〉 Modell nicht angepasst wird - kann dies zu einer Situation führen, in der ein jeder in ein 〈offizielles〉 Modell hinein sozialisiert wird, das von niemandem mehr ernst genommen wird» ${ }^{24}$.

Konkret bedeutet dies etwa, dass das Medium und die Inhalte der Bibel nicht einfach in alltagssprachliche Unkenntlichkeit transferiert werden, sondern gerade in ihrer Widerständigkeit in gebildete Kommunikation einzubringen sind.Texterschliessung nimmt folglich eine Art Leitplanken-Charakter an, insofern durch diese einerseits ein bestimmter gedanklicher Korridor eröffnet wird, andererseits der Spielraum aber auch begrenzt wird, um sowohl desorientierende Komplexitätsreduktion wie radikale Offenheitszumutung zu vermeiden. ${ }^{25}$

Aber auch Prozesse der ästhetischen Bildung sind hierbei in dem Horizont mitzudenken, dass das ästhetische Moment von Lehre, Lernen und Vermittlung in der Entfaltung differenter Wahrnehmungen besteht: "Gemeinschaft ist die Gemeinschaft der durch differente Wahrnehmung Verschiedenen ${ }^{26}$ - wobei gilt, dass sich erst anhand der Inhalte und unter Berücksichtigung der Rahmungen und Bedingungen vor Ort Formen für ästhetisch reflektierte Vermittlungsprozesse entwickeln lassen ${ }^{27}$.

Grundsätzlich ist in jedem Fall vorauszusetzen, dass für ein solches kognitives Verstehen wie ästhetisch ausgerichtetes Annähern im Modus gemeinsamer Konstruktionsprozesse die Motivation zum gemeinsamen Durchdenken des Eigen-Sinns biblischer Überlieferung ihrerseits in wortwörtlich an-sprechender Weise zu geschehen hat. Problematisch wäre es hingegen, würde man mit Hilfe der eigenen Angebots- und Kommunikationsstruktur lediglich den Fokussierungstendenzen auf individuelle Befindlichkeiten $\mathrm{zu}$ entsprechen suchen.Vielmehr ist Religion über ihre individuelle Seite hinaus auch in ihren Potentialen ethischer Weltbewältigung $\mathrm{zu}$ zeigen.

Insofern ist für die Bestimmung der Bildungsaufgabe von Religion etwa die nachordnende Unterscheidung zwischen einer ethisch ausgerichteten Orientierung an Schlüsselproblemen einer-

${ }^{24}$ Luckmann, Unsichtbare Religion, s. Anm. 9, 130.

${ }^{25}$ Vgl. G. Brenner, Bildung und Lernen. Anmerkungen zur Ambivalenz der Subjektorientierung, in: B. Hafeneger, Subjektdiagnosen. Subjekt, Modernisierung und Bildung, Schwalbach/Ts. 2005, 231.

${ }^{26}$ P. Maset, Ästhetische Bildung der Differenz. Kunst und Pädagogik im technischen Zeitalter, Stuttgart 1995, 110.

${ }^{27}$ Vgl. etwa A. Besand, Angst vor der Oberfläche. Zum Verhältnis ästhetischen und politischen Lernens im Zeitalter Neuer Medien, Schwalbach/Ts. 2004, 282. 
seits und der Fokussierung auf die "vorwiegend ästhetisch konturierten Bereiche des Zweck-losen, nicht Funktionalisierbaren, aber persönlich Bedeutsamen ${ }^{28}$ andererseits ebenso wenig sinnvoll wie die Priorisierung erfahrbarer Religion gegenüber der normativen Bedeutung traditioneller christlicher Gehalte. Vielmehr ist als Ziel religiöser Bildung der Erwerb einer religiösen Kompetenz anzusehen, die sich durch eine "Balance der verschiedenen kognitiven, emotionalen, kommunikativen und spirituellen Dimensionen von Religiosität» ${ }^{29}$ auszeichnet.

Dass dieser Versuch der inhaltsorientierten Versprachlichung und erst recht der ethischen Orientierung dazu führen kann, dass Jugendliche dies als sperrig empfinden und sich solchen Deutungsangeboten in aller Vehemenz widersetzen, ist aus dem guten Grund zu riskieren, der im riskanten unsichtbar-sichtbaren Inhalt der christlichen Botschaft selbst besteht. ${ }^{30}$

- Dr. Thomas Schlag ist Assistenzprofessor für Praktische Theologie mit den Schwerpunkten Religionspädagogik und Kybernetik an der Universtität Zürich.

\footnotetext{
${ }^{28}$ So Kunstmann, Religiosität und Bildung, s. Anm. 22, 169.

${ }^{29}$ U. Hemel, Religionsphilosophie und Philosophie der Religiosität. Ein Zugang über die Typologie religiöser Lebensstile, in: Angel u.a., Religiosität, s. Anm. 22, 112. ${ }^{30}$ Zur produktiven Bedeutung riskanter Kommunikation im kirchlichen Kontext vgl. J. Hermelink, Die Vielfalt der Mitgliedschaftsverhältnisse und die prekären Chancen der kirchlichen Organisation, in:W. Huber/J. Friedrich/P. Steinacker, Kirche in derVielfalt der Lebensbezüge. Die vierte EKD-Erhebung über Kirchenmitgliedschaft, Gütersloh 2006, $419 f$.
} 